\title{
Differential Impacts of Repeated Sampling on Odor Representations by Genetically-Defined Mitral and Tufted Cell Subpopulations in the Mouse Olfactory Bulb
}

\author{
(ㄱ)Thomas P. Eiting and ${ }^{(}$Matt Wachowiak \\ Department of Neurobiology and Anatomy, University of Utah, Salt Lake City, Utah 84103
}

Sniffing, the active control of breathing beyond passive respiration, is used by mammals to modulate olfactory sampling. Sniffing allows animals to make odor-guided decisions within $\sim 200 \mathrm{~ms}$, but animals routinely engage in bouts of high-frequency sniffing spanning several seconds; the impact of such repeated odorant sampling on odor representations remains unclear. We investigated this question in the mouse olfactory bulb (OB), where mitral and tufted cells (MTCs) form parallel output streams of odor information processing. To test the impact of repeated odorant sampling on MTC responses, we used two-photon imaging in anesthetized male and female mice to record activation of MTCs while precisely varying inhalation frequency. A combination of genetic targeting and viral expression of GCaMP6 reporters allowed us to access mitral cell (MC) and superficial tufted cell (sTC) subpopulations separately. We found that repeated odorant sampling differentially affected responses in MCs and sTCs, with MCs showing more diversity than sTCs over the same time period. Impacts of repeated sampling among MCs included both increases and decreases in excitation, as well as changes in response polarity. Response patterns across simultaneously-imaged MCs reformatted over time, with representations of different odorants becoming more distinct. Individual MCs responded differentially to changes in inhalation frequency, whereas sTC responses were more uniform over time and across frequency. Our results support the idea that MCs and TCs comprise functionally distinct pathways for odor information processing, and suggest that the reformatting of MC odor representations by high-frequency sniffing may serve to enhance the discrimination of similar odors.

Key words: coding; in vivo; mitral cell; odor; sniffing; two-photon imaging

\section{Significance Statement}

Repeated sampling of odorants during high-frequency respiration (sniffing) is a hallmark of active odorant sampling by mammals; however, the adaptive function of this behavior remains unclear. We found distinct effects of repeated sampling on odor representations carried by the two main output channels from the mouse olfactory bulb (OB), mitral and tufted cells (MTCs). Mitral cells (MCs) showed more diverse changes in response patterns over time as compared with tufted cells (TCs), leading to odorant representations that were more distinct after repeated sampling. These results support the idea that MTCs contribute different aspects to encoding odor information, and they indicate that MCs (but not TCs) may play a primary role in the modulation of olfactory processing by sampling behavior.

Received Feb. 3, 2020; revised June 16, 2020; accepted June 18, 2020.

Author contributions: T.P.E. and M.W. designed research; T.P.E. performed research; T.P.E. and M.W. analyzed data; T.P.E. and M.W. wrote the paper.

This work was supported by National Institutes of Health Grants F32DC015389 (to T.P.E.) and DC006441 (to M.W.).

The authors declare no competing financial interests.

We thank S. Burton, A. Moran, S. Short, and I. Youngstrom for helpful comments, G. Vasquez for help with virus injections, and J. Ball and R. Kummer for help with animal husbandry and histological processing. We also thank two anonymous reviewers, whose constructive feedback improved this paper substantially.

Correspondence should be addressed to Thomas P. Eiting at tom.eiting@utah.edu.

https://doi.org/10.1523/JNEUROSCI.0258-20.2020

Copyright $\odot 2020$ the authors

\section{Introduction}

Olfaction, a key sensory modality for mammals, is an important model system for understanding how active sensation contributes to sensory processing (Uchida et al., 2006; Wachowiak, 2011; Jordan et al., 2018b). Sniffing, the active control of breathing beyond passive respiration, is used by mammals to modulate olfactory sampling. Sniffing provides temporal control over olfactory sensation and can increase access to odor information by increasing the flow of odorants through the nasal cavity, and also by allowing multiple samples of odorant in a shorter time (Zhao et al., 2006; Verhagen et al., 2007; Courtiol et al., 2011; Rygg et al., 2017). Repeated high-frequency $(3-10 \mathrm{~Hz})$ inhalations are a hallmark of sniffing behavior in most mammals, including 
rodents (Youngentob et al., 1987; Laska, 1990; Thesen et al., 1993; Wesson et al., 2008a). While mice and rats can discriminate odors in as little as one sniff (150-200 ms; Uchida and Mainen, 2003; Abraham et al., 2004), they frequently exhibit longer sniffing bouts lasting several seconds (Welker, 1964; Wesson et al., 2008a). How repeated sampling influences olfactory processing by neural circuits in the olfactory bulb (OB) or elsewhere is not well understood.

Previous work has shown that rapid sniffing induces adaptation of olfactory sensory neuron (OSN) inputs to the OB (Verhagen et al., 2007); more recently, we and others have found that inhalation frequency modulates subthreshold membrane potential and spiking patterns in mitral and tufted cells (MTCs), the two major subclasses of $\mathrm{OB}$ output neurons (Bathellier et al., 2008; Carey and Wachowiak, 2011; Díaz-Quesada et al., 2018; Jordan et al., 2018b). Multiple functions of this frequency-dependent modulation have been proposed, including enhancing olfactory sensitivity, increasing the salience of odorants against a background, and improving fine-scale odor discrimination.

There is substantial evidence that mitral cells (MCs) and tufted cells (TCs) have distinct odorant response properties and likely contribute differentially to encoding odor information (Nagayama et al., 2004; Griff et al., 2008; Fukunaga et al., 2012; Jordan et al., 2018a; Short and Wachowiak, 2019). Putative MTCs also respond to odorants differently during brief bouts of high-frequency sniffing (Jordan et al., 2018b). However, how, or whether, these populations differentially represent odorants during repeated sampling of odorants remains unclear. Ultimately, because odor coding involves combinatorial activity patterns across many cells, understanding how sniffing impacts odor representations requires recording from multiple cells simultaneously, ideally using genetic and anatomic criteria to define subpopulations independent of their functional properties.

Here, we addressed these knowledge gaps by imaging from genetically- and anatomically-defined subsets of MCs and TCs during repeated odorant sampling at different inhalation frequencies. We use an artificial inhalation paradigm in anesthetized mice to allow for precise reproduction of odorant sampling regimes and comparison of responses across cell type subpopulations, with minimal impact of centrifugal inputs or other behavioral state-dependent factors. We found substantial population-level differences between MCs and a subset of TCs defined by their superficial location and their expression of the peptide transmitter cholecystokinin (CCK). The $\mathrm{CCK}^{+}$superficial TC (sTC) population showed stronger coupling to individual inhalations, few suppressive responses, and little change in its population response pattern during repeated odorant sampling. In contrast, MCs showed diverse changes in excitation and suppression with repeated odorant sampling, such that the MC population response pattern reformatted substantially from the beginning to the end of the odorant presentation, and did so in a frequency-dependent manner. These effects point to an important role for inhalation frequency-dependence in odor perception and differential contributions by MC and sTC populations in encoding odor information over repeated samples.

\section{Materials and Methods}

\section{Animals}

Adult transgenic mice (ages 6-16 weeks) of both sexes ( $n=12$ males and 13 females) were used in all experiments. Imaging in mice was conducted in Cck-IRES-cre [The Jackson Laboratory (Jax) stock \#012706; Taniguchi et al., 2011] mice for imaging in sTCs or in either Pcdh21-cre (Mutant Mouse Resource and Research Center stock \#030952-UCD;
Gong et al., 2003) mice or Tbet-cre (Jax stock \#024507; Haddad et al., 2013) mice for imaging in MCs. Mice were either crossed with Ai95 (RCL-GCaMP6f)-D (Jax stock \#024105; Madisen et al., 2015) reporter mice or injected with adeno-associated viral vectors (AAV) to drive expression. All procedures were conducted according to NIH guidelines and were approved by the Institutional Animal Care and Use Committee of the University of Utah.

\section{Viral vector expression}

GCaMP6f/s expression was achieved using recombinant viral vectors, AAV1, AAV5, or AAV9 serotypes of hSyn.Flex.GCaMP6f.WPRE.SV40 or hSyn.Flex.GCaMP6s.WPRE.SV40 (UPenn Vector Core). Virus titers were as follows: $1.7 \times 1012$ to $1.4 \times 1013$ (GCaMP6f) or $7.0 \times 1012$ to $7.3 \times 1013$ (GCaMP6s). Virus was injected using pulled glass pipettes into the anterior piriform cortex (aPC) at the following coordinates (relative to bregma): $2.4 \mathrm{~mm}$ anterior, $1.6 \mathrm{~mm}$ lateral, and $3.5 \mathrm{~mm}$ ventral. Injections were performed as described previously (Rothermel et al., 2013; Wachowiak et al., 2013), achieving an infection rate of $\sim 80 \%$ as employed by our lab (Rothermel et al., 2013). Animals were injected with carprofen $(5 \mathrm{mg} / \mathrm{kg}$; analgesic) and enrofloxacin $(10 \mathrm{mg} / \mathrm{kg}$; antibiotic) immediately before surgery as well as the following day. After surgery, animals were singly-housed and were allowed to recover on a heating pad before transfer back to the animal colony. Imaging experiments occurred 14-45 $\mathrm{d}$ after injection.

\section{Olfactometry}

Odorants were delivered via an air-dilution olfactometer, in which the odorants were first diluted in mineral oil, followed by an air-phase dilution, for a final estimated concentration presented to the animal of 10-20 ppm (Wachowiak et al., 2013; Economo et al., 2016). Odorants of different chemical groups (esters, aldehydes, ketones, organic acids) were presented in random order with respect to inhalation frequency, and repeated for eight trials (six trials in two sTC glomerular experiments), with a minimum $36 \mathrm{~s}$ intertrial interval (ITI). Inhalation (1, 3 , and $5 \mathrm{~Hz}$ ) was controlled using an artificial inhalation paradigm (Wachowiak and Cohen, 2001; Díaz-Quesada et al., 2018; Eiting and Wachowiak, 2018). Stimuli and artificial inhalation were controlled with Labview software (National Instruments).

\section{In vivo two-photon imaging}

Animals were initially anesthetized with pentobarbital $(50 \mathrm{mg} / \mathrm{kg})$; and long-term anesthesia was maintained with isoflurane $(0.5-1.0 \%$ in oxygen) for the duration of the experiment. Body temperature was maintained at $37^{\circ} \mathrm{C}$. Following a craniotomy $(\sim 1.5 \times 1 \mathrm{~mm})$ over one $\mathrm{OB}$, we imaged glomeruli or cell bodies at appropriate depth: $20-30 \mu \mathrm{m}$ below olfactory nerve layer (ONL) for glomeruli, $\sim 120-150 \mu \mathrm{m}$ below the ONL for sTCs and $\sim 240-270 \mu \mathrm{m}$ below ONL for MCs. Calcium signals (GCaMP6f/6s) were collected using a two-photon laser and microscope system (Neurolabware), running a Ti-Sapphire laser (Coherent, Chameleon Ultra-II) operating at $920-940 \mathrm{~nm}$. Imaging was performed through a $16 \times, 0.8 \mathrm{NA}$ objective (Olympus), and emitted light was collected through a GaAsP photomultiplier tube (Hamamatsu, H10770B40). Frame rate was $15.5 \mathrm{~Hz}$.

\section{Data analysis}

Image processing and analysis of optical signals followed procedures similar to those described previously (Wachowiak et al., 2013; Economo et al., 2016). Analyses of calcium data were performed on fluorescence signals collected at $15.5 \mathrm{~Hz}$. Regions of interest (ROIs) were either glomeruli or cell bodies, selected as contiguous polygons of responsive pixels. ROIs were initially defined based on visually inspecting average odor-evoked response maps and choosing a single polygon above a certain visual threshold of response relative to background. In cases where cell bodies and dendrites appeared to overlap, we selected and analyzed only those portions of the cell body that were non-overlapping. Some ROIs were selected using a manual procedure to select strong responses and when the automated thresholding procedure failed to select single cell bodies or glomeruli. $\Delta \mathrm{F} / \mathrm{F}$ values were calculated as the change in fluorescence during $8 \mathrm{~s}$ odorant stimulation compared with the $4 \mathrm{~s}$ 
A

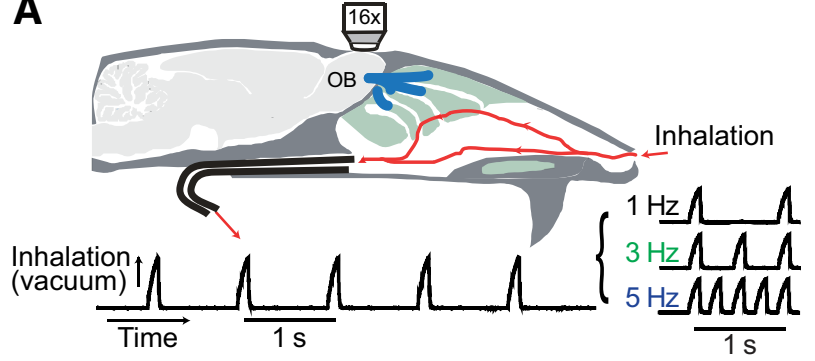

B
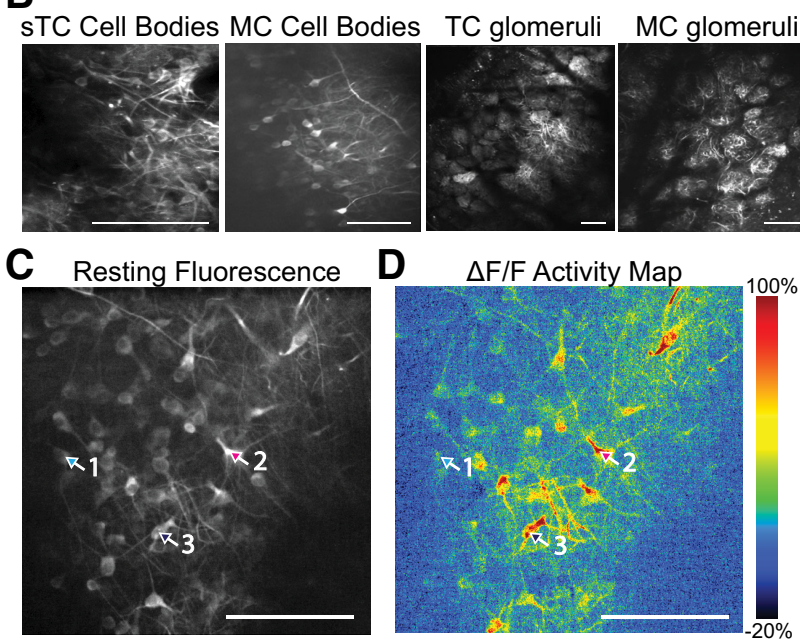

$\mathbf{E}_{\text {odor }}$

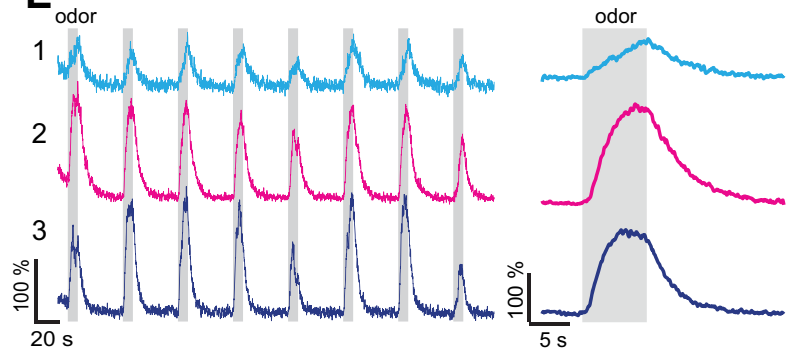

Figure 1. Imaging MTC population odor responses with artificial inhalation. $\boldsymbol{A}$, Schematic of experimental paradigm. Odorants are delivered using an artificial inhalation paradigm, with inhalation frequencies of 1,3 , or $5 \mathrm{~Hz}$. A sample inhalation trace $(1 \mathrm{~Hz})$ is pictured at the bottom. $\boldsymbol{B}$, Representative images of resting fluorescence in three types of experiments, showing typical fields of view for sTC cell bodies, MC cell bodies, TC glomeruli, and MC-innervated glomeruli. C, Resting fluorescence image from one FOV, showing over $50 \mathrm{MC}$ cell bodies. $D, 0$ dorant-evoked calcium signals, represented as $\Delta F / F$, of the same FOV shown in $\boldsymbol{C}$. $\boldsymbol{E}$, left, Example traces (from ROls indicated in $\boldsymbol{C}, \boldsymbol{D}$ ), showing evoked fluorescence for eight consecutive trials in which odorant is presented during $5 \mathrm{~Hz}$ inhalation for $8 \mathrm{~s}$ per trial. Right, Average responses of the three ROls. For most analyses, average responses were used in calculations. All scale bars $=100 \mu \mathrm{m}$.

immediately before odorant stimulation, divided by the mean baseline fluorescence value. ROIs were included if the peak value of their odorevoked $\Delta \mathrm{F} / \mathrm{F}$ signal exceeded $6 \mathrm{SDs}$ of the signal during the $4 \mathrm{~s}$ immediately before the odorant stimulation. If a cell or glomerulus was responsive at one frequency but not another, it was kept and analyzed across all three inhalation conditions. In this way, a non-response can be reasonably interpreted as contributing to the signal. Analyses were conducted only when blank air generated few or no responses (max $5 \%$ responding cells/glomeruli), and individual ROIs were removed from analyses if they responded to blank air stimulation. Correlationto-early calculations were conducted as follows. Data were broken into matrices of ROI-by-time fluorescence values for each odorant-by-frequency $(1,3$, or $5 \mathrm{~Hz})$ combination. ROI signals were averaged over each $1 \mathrm{~s}$ time period ( $\sim 15$ time bins of data), for the full $8 \mathrm{~s}$ odorant presentation. The ROI population response at a given $1 \mathrm{~s}$ time point was correlated with the response in the $1 \mathrm{~s}$ immediately prior, with the correlation at the starting time bin set to "1." These correlations were then grouped together either by experiment or by cell type, as indicated in Results.

The analysis in which we broke the MC-cell body dataset into groups of cells with different response strengths was done as follows for each inhalation frequency and odor combination. First, for each odorant response, the $\mathrm{MC}$ population was split into three groups as follows: strongly excitatory (the top $50 \%$ of all excitatory responses), weakly excitatory (the bottom $50 \%$ of all excitatory responses), and suppressed cell groups. This resulted in groups of 4-22 cells in each odor-by-frequency case for a given field of view (FOV; average 7-11 cells across all FOVs), save for two experiments, in which $5 \mathrm{~Hz}$ suppressive responses were not observed. Finally, the cells that made up each group were subtracted from the overall populations, and correlations-over-time were recalculated as above.

Principal components analysis (PCA) was conducted for experiments in which three or more odorants generated consistent responses, resulting in five MC-cell body ( $n=13-58$ ROIs) experiments and three sTC-cell body ( $n=18-23$ ROIs) experiments. For each experiment, PCA was conducted as follows. First, responses to any given odor-by-inhalation frequency condition were averaged across the eight repeated presentations of that stimulus, and binned per frame (i.e., $\sim 15.5 \mathrm{~Hz}$; each bin $\sim 65 \mathrm{~ms}$ ). Data were then concatenated for all odors into one dataset for each inhalation-frequency condition. PCA was conducted on each of these three datasets $(1,3$, and $5 \mathrm{~Hz}$ ), computed on ROI-by-time (twophoton sampling rate) matrices, similar to what has been done in previous studies (Bathellier et al., 2008). The PCAs were primarily used for visualization.

Finally, Euclidean distances (calculated as the square root of the sum of squared distances) were calculated across cell responses between each successive time bin, and these were summed across all odors for each inhalation condition. Euclidean distances were standardized by number of odorants within a given experiment, because this measure provided an unbiased method to compare the spread of points in visualization (PC) space across experiments or conditions. These values were used as a way to examine how the temporal evolution of MC and sTC odorant responses were represented in the cell body populations.

\section{Experimental design and statistical analyses}

Unless otherwise noted, analyses were done using mean responses within a given experimental preparation as our unit of replication. Statistical tests were conducted using custom scripts written in MATLAB 2019b (MathWorks) or R v.3.3.2 (R Foundation for Statistical Computing). Details of each analysis are presented in the relevant section of the results. Raw data and analysis code are available on request.

\section{Results}

Our major goals were to understand how inhalation frequency affects activity patterns of MTC populations, and to explore differences in their responses in the context of inhalation frequency. To achieve these aims we collected fluorescent calcium signals from MCs and sTCs under two-photon microscopy using an artificial-inhalation paradigm to control inhalation frequency in isoflurane-anesthetized mice (Fig. 1A,B; Díaz-Quesada et al., 2018; Eiting and Wachowiak, 2018). To target MCs, protocadherin21-Cre mice were injected with cre-dependent virus into the aPC to generate expression predominately in piriform-projecting MCs (Rothermel et al., 2013). sTCs were targeted by crossing CCK-IRES-Cre mice with Rosa-GCaMP6f mice (line Ai95D), as previously reported (Economo et al., 2016; Short and Wachowiak, 2019). For each cell type, we lowered the imaging plane to the appropriate depth. We gathered MC and sTC responses from populations of cells imaged while presenting clean air and then odorant at different inhalation frequencies (see Materials and Methods). We presented eight successive odorant presentations of $8 \mathrm{~s}$ duration (interspersed with a $36 \mathrm{~s}$ 
ITI) at each inhalation frequency. Example resting fluorescence, activity map, and traces from a MC-cell body imaging experiment show examples of average traces produced from individual trials (Fig. $1 C-E$ ). In general, variability from trial to trial was small and, as is apparent in Figure $1 E$, tended to be correlated across cells, suggesting a difference in stimulation effectiveness or other global change in excitability rather than variations in cell-specific responses.

We first compared responses of MCs and sTCs to repeated sampling of odorant at 1,3 , and $5 \mathrm{~Hz}$. Examples of imaging experiments targeted to each cell type are shown in Figure $2 A, B$, illustrating qualitative differences in how MCs and sTCs respond to repeated sampling, and how these responses change as a function of frequency. MCs showed more diverse temporal responses over time that included slower increases in activity peaking over the course of odorant presentation, while sTC responses tended to be simpler, generally following the onset and offset of odorant presentation (Fig. 2A,B). sTCs also more commonly followed individual inhalations at $1 \mathrm{~Hz}$ than did MCs. These trends were evident across the entire population of imaged MCs and sTCs, compiled from seven and five mice, respectively (Fig. 2C,D). Finally, suppressive responses were common in MCs but were rarely observed in sTCs. MC responses included a substantial percentage of suppressive cells: 207/655 (32\%) cell-odor pairs at $1 \mathrm{~Hz}, 163 / 655$ (25\%) cell-odor pairs at $3 \mathrm{~Hz}$, and 168/655 (26\%) cell-odor pairs at $5 \mathrm{~Hz}$ were suppressive. By contrast there were very few suppressive sTC responses: $23 / 445$ (5\%) cell-odor pairs at $1 \mathrm{~Hz}, 31 / 445(7 \%)$ cell-odors pairs at $3 \mathrm{~Hz}$, and $24 / 445(5 \%)$ cell-odor pairs at $5 \mathrm{~Hz}$ were suppressive. Signals from MC cell bodies using both GCaMP6f (four mice) and GCaMP6s (three mice) showed similar temporal response patterns and prevalence of suppression (not shown), suggesting that the observed differences between MC and sTC populations were likely not due to differences in the temporal dynamics of the calcium indicator used. Overall, this suite of differences recapitulates those reported earlier at $2 \mathrm{~Hz}$ inhalation frequency (Economo et al., 2016).

MCs responded heterogeneously to different inhalation frequencies (Fig. 2A). For example, cell 3 in Figure $2 A$ shows no response at $1 \mathrm{~Hz}$ inhalation but strong excitation at $5 \mathrm{~Hz}$, while cell four switches response polarity from excitation at 1 and $3 \mathrm{~Hz}$ to suppression at $5 \mathrm{~Hz}$. Neighboring cells also show different responses to repeated sampling of the same odorant. For example, cell 3 in Figure $2 A$ (mentioned above) can be contrasted with its neighbor, cell 5 , which responds fairly consistently across all three inhalation frequencies. Across the MC population, cells switched response polarity from suppressed to excited roughly three to four times as frequently as excited to suppressed: $49 / 438$ (11\%) excited to suppressed from 1 to $3 \mathrm{~Hz}, 64 / 438$ (15\%) excited to suppressed from 1 to $5 \mathrm{~Hz}, 84 / 217$ (39\%) suppressed to excited from 1 to $3 \mathrm{~Hz}$, and 111/217 (51\%) suppressed to excited from 1 to $5 \mathrm{~Hz}$ (Fig. 2C). MCs also became more excitable with increasing inhalation frequencies: more cells responded to greater numbers of odorants at 3 and $5 \mathrm{~Hz}$ compared with $1 \mathrm{~Hz}$ (Fig. 2D). By contrast, sTCs typically responded similarly to all three inhalation frequencies (Fig. $2 B, E$ ), although more sTCs responded to greater numbers of odorants at higher frequencies. In many cells a decrease in excitation suggesting adaptation was apparent with repeated sampling at higher frequencies (Fig. $2 B$, cells 4 and 5). Changes in response polarity in sTCs were much rarer compared with MCs: 22/402 (5\%) switched from excited to suppressed responses when comparing $1-3 \mathrm{~Hz}$, and 17/402 (4\%) switched from excited to suppressed in the 1 to $5 \mathrm{~Hz}$ case.
Furthermore, while only $5-7 \%$ of sTCs showed suppressive responses to $1 \mathrm{~Hz}$ inhalation, nearly half of these cells switched polarity from suppression to excitation when inhalation frequency increased to $3 \mathrm{~Hz}$ (39\%; 9/23 cells) or $5 \mathrm{~Hz}$ (61\%; 14/23 cells). These differences between MCs and sTCs were likely not due to differences in frequency-dependent excitability, as sTCs showed a similar pattern to MCs of cells become more excitable with increasing inhalation frequency (Fig. 2F). Overall, these analyses indicate that MCs and sTCs show distinct impacts of inhalation frequency on responses to repeated odorant inhalation, but that across both cell types, as inhalation frequency increased, cells tended to switch from suppression to excitation much more frequently than from excitation to suppression.

Heterogeneity in MC (and to a lesser extent sTC) responses implies that neural representations of odor identity, measured in terms of spike rate across each population, will change as a function of repeated odor sampling (Patterson et al., 2013; DíazQuesada et al., 2018). Example experimental preparations in Figure $3 A, B$ show how populations of cells [MCs (Fig. 3A) and sTCs (Fig. $3 B$ )] change over an $8 \mathrm{~s}$ odorant presentation at 1 and $5 \mathrm{~Hz}$ inhalation. In the MC example, numerous cell bodies changed their response polarity or their response amplitude across the $8 \mathrm{~s}$ odorant period, and this shift occurred more often at higher inhalation frequency (Fig. $3 A$ ). By contrast, in the sTC example, very few cells switched their response polarity, or even changed their activity level appreciably, regardless of inhalation frequency (Fig. 3B).

To quantify changes among populations of MCs and sTCs, we correlated the population response to an odorant at a given time point to the population response at the first time point (corresponding to the first inhalation at $1 \mathrm{~Hz}$; for precise times, see Materials and Methods) over the length of odorant presentation. This analysis was performed separately for each ensemble of MCs or sTCs imaged in the same FOV $(n=13-58$ MCs and 1043 sTCs per FOV, one FOV per animal). Correlation series for different odorants tested in the same FOV were averaged before compiling across animals, to avoid bias from different numbers of odorants tested in different animals. These analyses revealed substantial differences in the evolution of odor representations between MCs and sTCs. Among MCs (Fig. 3C), high-frequency inhalation appeared to result in more substantial decorrelation compared with low-frequency inhalation: after $8 \mathrm{~s}$, the correlation to the initial response pattern was relatively high at $1 \mathrm{~Hz}$ $(r=0.72 \pm 0.29$, mean $\pm \mathrm{SD})$, with lower correlation values at higher inhalation frequencies $(3 \mathrm{~Hz}, r=0.63 \pm 0.37 ; 5 \mathrm{~Hz}$, $r=0.61 \pm 0.37)$. These differences across frequency were not statistically significant, however $(n=7$ mice, Mann-Whitney $U$ test, 1 vs $3 \mathrm{~Hz}, p=0.30 ; 1$ vs $5 \mathrm{~Hz}, p=0.71$ ), due to the relative high variance in the degree of decorrelation for different fields of view (see below). Nonetheless this result for MCs contrasts with that for sTCs (Fig. 3D), which maintained highly similar response patterns over the 8-s odor duration, with correlations to the initial response of $r=0.90 \pm 0.07$ in the $1 \mathrm{~Hz}$ case, $0.89 \pm 0.02$ at $3 \mathrm{~Hz}$, and $0.81 \pm 0.12$ at $5 \mathrm{~Hz}(n=5$ mice $)$. Regardless of inhalation frequency, MCs decorrelated significantly more over the $8 \mathrm{~s}$ compared with sTCs (correlation coefficients in final time bin for all frequencies, two sample $t$ test, $\left.p=9.2 \times 10^{-5}\right)$. MC cell bodies decorrelated to similar levels when either GCaMP6s or GCaMP6f was used (Fig. 3C), indicating that the differences observed between MC and sTC populations were not a result of different GCaMP reporters; in subsequent analyses, we combined data from both variants. Overall, this analysis shows that MC responses show more 

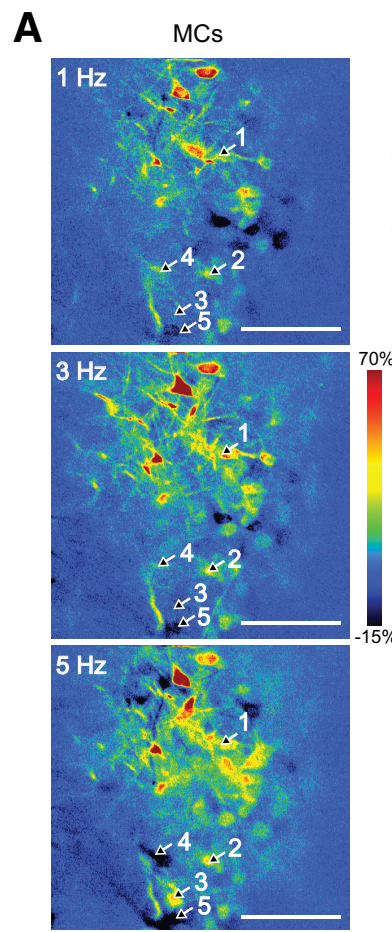

C

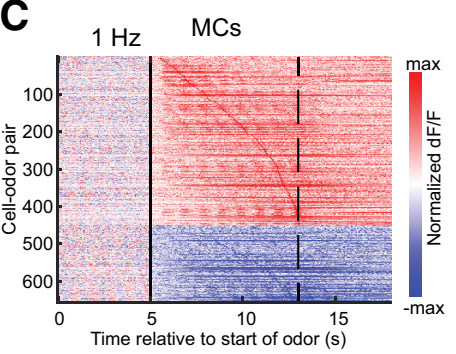

$3 \mathrm{~Hz}$

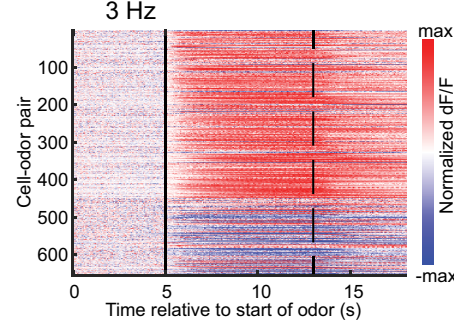

$5 \mathrm{~Hz}$

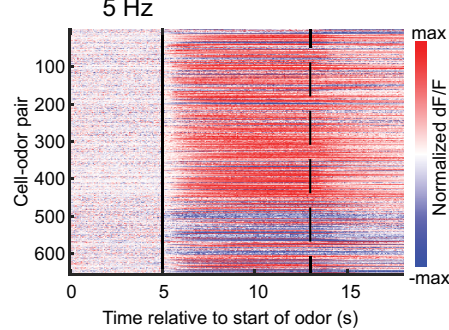

B
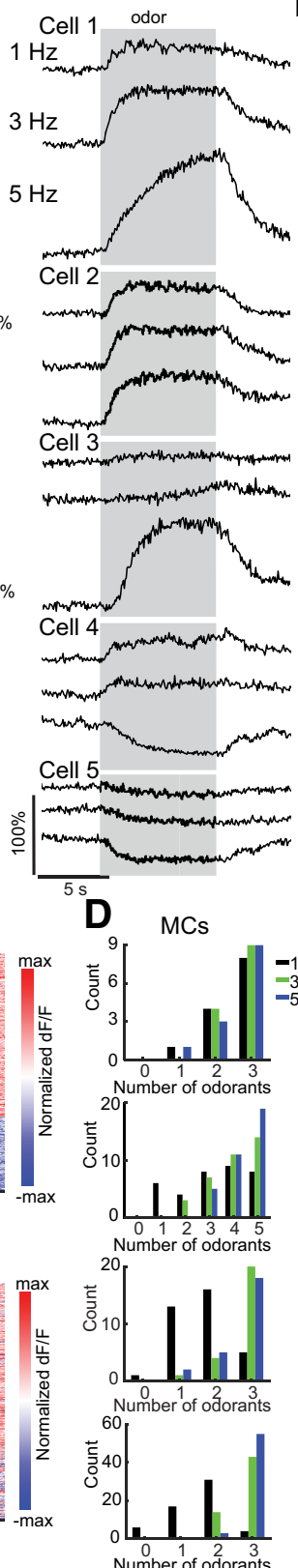$$
40
$$

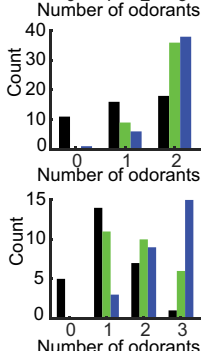

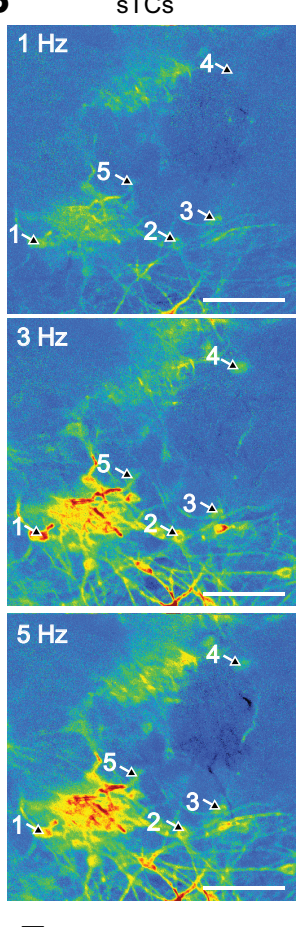

E

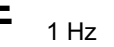

sTCs

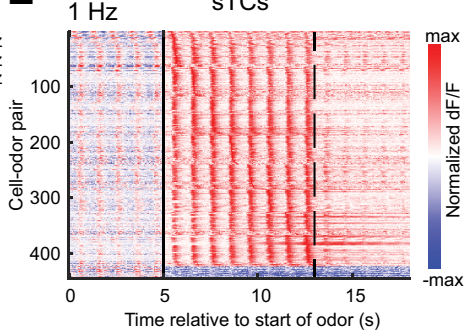

r sTCs

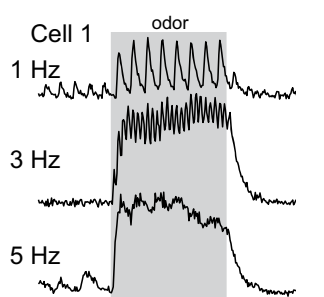

$200 \%$

Cell 2 MMMMM

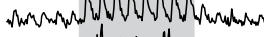

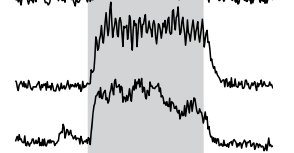

Cell 3 MNMWMM
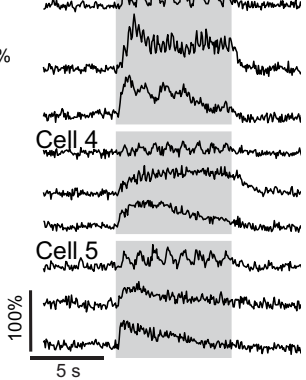

Cell 5 mararas

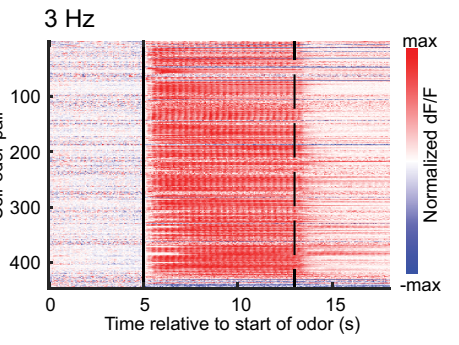

$5 \mathrm{~Hz}$

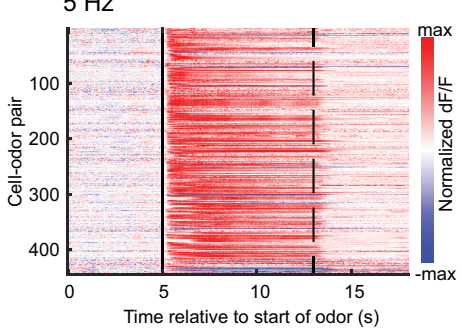

Figure 2. Odorant-evoked activity varies with inhalation frequency. $\boldsymbol{A}$, Example trial-averaged responses from five $\mathrm{MC}$ cell bodies, showing diverse responses to ethyl butyrate (0.1\%) at three different inhalation frequencies. $\boldsymbol{B}$, Trial-averaged responses from five sTC cell bodies, showing a relative lack of diversity in temporal responses among cells within a given preparation. In all cells, fluorescence signals clearly follow $1 \mathrm{~Hz}$ inhalations (top rows for each cell), but this effect disappears at higher frequencies. Scale bars for the maps in $\boldsymbol{A}, \boldsymbol{B}=100 \mathrm{\mu m}$. $\boldsymbol{C}$, Time-by-activity plots for all MC cell-odor pairs, rank-ordered by time-to-peak activity at $1 \mathrm{~Hz}$. MC responses are diverse and prolonged, and activity patterns change substantially among cells at higher inhalation frequency. D, Cell-response histograms for six MC experiments, showing histograms of how many odorants each cell responded to, across all three inhalation frequencies. For most experiments, more cells responded to higher numbers of odorants at elevated inhalation frequencies ( $3 \mathrm{and} 5 \mathrm{~Hz}$ ). At $1 \mathrm{~Hz}$ (black bars), usually many more cells responded to fewer numbers of odorants. $\boldsymbol{E}$, Time-by-activity plot for sTC cell bodies. Note that, compared with $\boldsymbol{C}$, peak sTC activity tends to occur earlier than peak MC activity, and sTC responses generally turned off quickly after odor presentation compared with MC responses. In both $\boldsymbol{C}, \boldsymbol{E}$, odorant offset is at $8 \mathrm{~s}$. $\boldsymbol{F}$, Cell-response histograms for three sTC experiments, similar to $\boldsymbol{D}$, again showing that more cells responded to greater number of odorants at higher inhalation frequencies. 
decorrelation in response patterns with repeated sampling than do sTCs, and suggests that higher inhalation frequencies may drive greater decorrelation in the MC population.

Linear decorrelation in population response patterns could arise from systematic changes in MC (or sTC) responsiveness. Such changes could include, for example, increases in excitation that saturate at the high end of a cell's dynamic range or rise above threshold at the low end, or decreases in excitation that have the opposite effects. Alternatively, decorrelation could result from distinct effects of repeated sampling in different cells. To distinguish between these possibilities, we measured the change in MC or sTC excitation over the course of the odorant presentation, expressed as the change in $\Delta \mathrm{F} / \mathrm{F}$ from the first to the last second of the presentation (Fig. 4A). There was substantial heterogeneity in the effect of repeated sampling on excitation, reflected as a large variance across cells in the change in $\Delta \mathrm{F} / \mathrm{F}$ (Fig. $4 A$ ). Across all cells analyzed and all inhalation frequencies, there was a significant net increase in $\Delta \mathrm{F} / \mathrm{F}$ from the first to the last second, although the magnitude of the difference was small relative to the variance across different cells (Wilcoxon sign-ranked test: $\mathrm{MC}, 1 \mathrm{~Hz}$, mean change in $\Delta \mathrm{F} / \mathrm{F}$ $\pm \mathrm{SD}=0.075 \pm 0.22, p=1.2 \times 10^{-11}$; $3 \mathrm{~Hz}, \quad 0.13 \pm 0.31, \quad p=1.1 \times 10^{-15}$; $5 \mathrm{~Hz}, \quad 0.12 \pm 0.36, \quad p=3.1 \times 10^{-10}$; sTC, $1 \mathrm{~Hz}, 0.12 \pm 0.31, p=7.5 \times$ $10^{-11} ; 3 \mathrm{~Hz}, 0.21 \pm 0.30, p=3.5 \times$ $10^{-26} ; 5 \mathrm{~Hz}, 0.056 \pm 0.29, p=3.1$ $\times 10^{-2}$; Fig. $4 A$ ). The magnitude of these differences did not vary significantly across inhalation frequencies among MCs, although it did among sTCs (Kruskal-Wallis test: MC, $\chi^{2}=$ $5.1_{2,1176}, p=0.08$; sTC, $\chi^{2}=49.8_{2,813}$, $\left.p=1.5 \times 10^{-11}\right)$, with the sTC responses at $3 \mathrm{~Hz}$ increasing significantly more than at 1 or $5 \mathrm{~Hz}$ inhalation frequencies (Bonferroni-adjusted pairwise comparisons, $p<0.00001$ for both $1 \mathrm{vs} 3 \mathrm{~Hz}$ and $3 \mathrm{vs} 5 \mathrm{~Hz}$ ). These results are consistent with the idea that cells respond to repeated sampling with a net increase in excitability over the duration of the odorant presentation.

We next asked whether increasing inhalation frequency systematically impacted MC or sTC excitability within a given cell, either initially (i.e., in the first second) or after prolonged sampling (i.e., after $8 \mathrm{~s})$. To test this, we compared response magnitude $(\Delta \mathrm{F} / \mathrm{F})$ for the same cell-odor pairs at $1 \mathrm{~Hz}$ inhalation with that at 3 and $5 \mathrm{~Hz}$, for both the initial (i.e., "early") and 8 s (i.e., "late") time points (Fig. 4B). We found that increasing inhalation
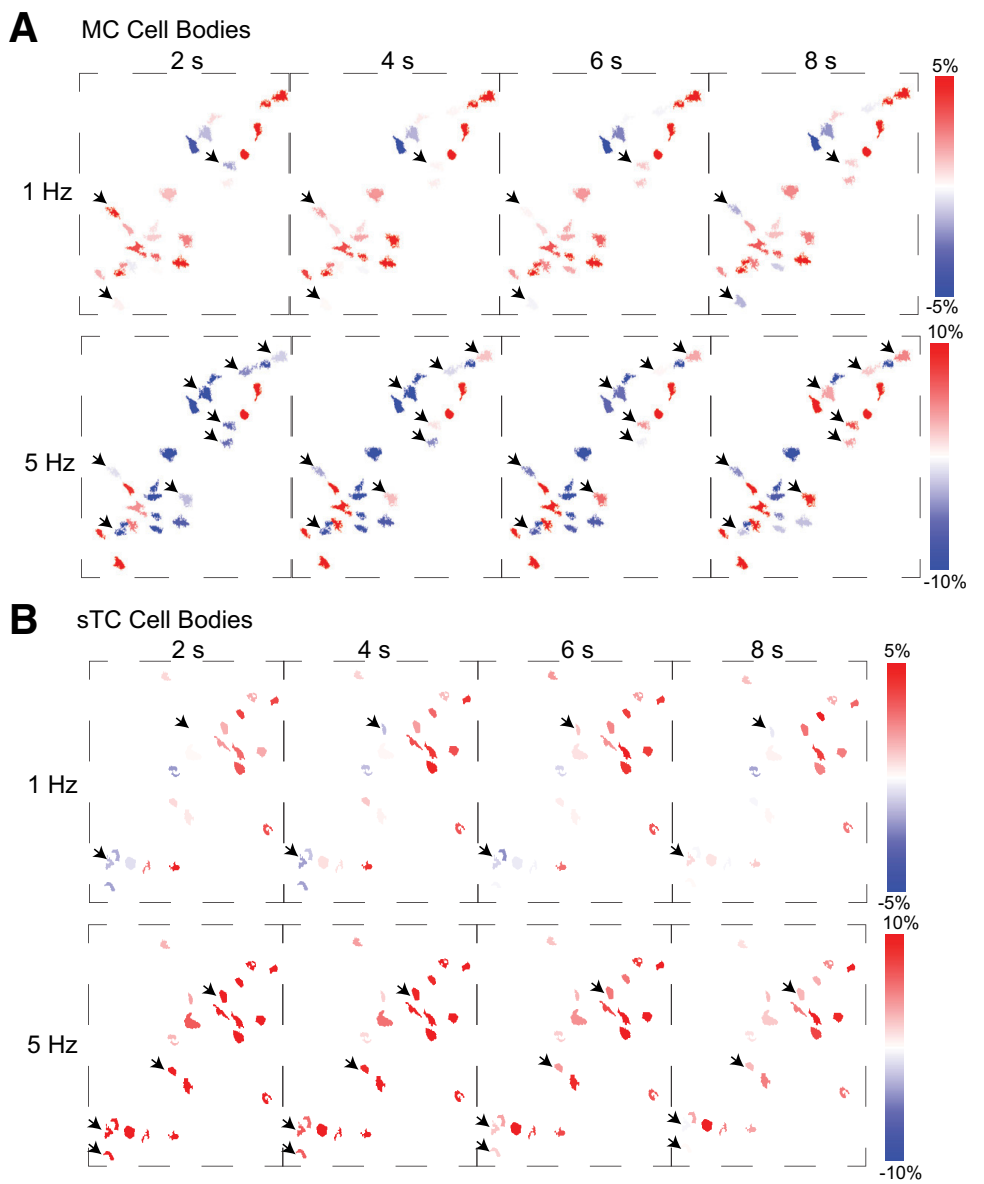

C MC Cell Bodies

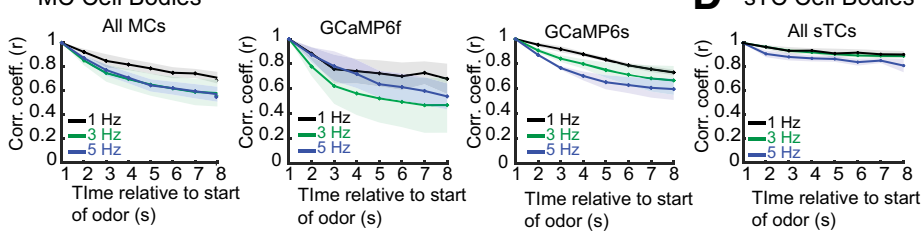

Figure 3. MC population responses evolve with repeated odorant sampling more than sTC populations. $\boldsymbol{A}$, Example MC-cell body experiment, showing responsive cells during 1 and $5 \mathrm{~Hz}$ inhalation at different time points. Time progresses horizontally in 2-s increments that represent the response maps during odorant presentation, averaged over $1 \mathrm{~s}$. A number of MC cell bodies change their response strength or response polarity during $5 \mathrm{~Hz}$ inhalation, with fewer changing during $1 \mathrm{~Hz}$ inhalation. Black arrows highlight cells whose response changes substantially or switches polarity during odor presentation. $\boldsymbol{B}$, Example sTC cell body experiment, displayed as in $\boldsymbol{A}$, showing a relative lack of changes in responses among individual cells, regardless of inhalation frequency. $\boldsymbol{C}$, over the length of odorant presentation. Correlations decreased faster at higher frequencies, although this difference was not statistically significant. Left, All MC-cell body data. Middle, Data from GCaMP6f experiments. Right, Data from GCaMP6s experiments. D, Plot of same analysis for sTC responses. Temporal decorrelation does not vary substantially across inhalation frequencies among sTCS. Decorrelation values differed between MCs and sTCs (see text for details). In both $\boldsymbol{C}, \boldsymbol{D}$, shaded areas represent SEM.

frequency significantly increased excitation for both early and late time points in both MCs and sTCs (Wilcoxon sign-ranked test: early $\mathrm{MC}: 3 \mathrm{~Hz}$, mean change in $\Delta \mathrm{F} / \mathrm{F} \pm \mathrm{SD}:=$ $0.024 \pm 0.067, p=1.2 \times 10^{-11} ; 5 \mathrm{~Hz}=0.023 \pm 0.078, p=3.0 \times$ $10^{-8}$; late MC: $3 \mathrm{~Hz}, 0.081 \pm 0.31, p=1.1 \times 10^{-9} ; 5 \mathrm{~Hz}=$ $0.072 \pm 0.4103, p=7.9 \times 10^{-3}$; early sTC: $3 \mathrm{~Hz}=0.17 \pm 0.15$, $p=2.6 \times 10^{-40} ; 5 \mathrm{~Hz}=0.19 \pm 0.25, p=2.6 \times 10^{-27}$; late sTC: $3 \mathrm{~Hz}=0.26 \pm 0.32, p=3.4 \times 10^{-27} ; 5 \mathrm{~Hz}=0.13 \pm 0.43, p=5.6 \times$ $10^{-9}$; Fig. $4 B$ ). The net increase in excitation in both the initial and late time bins was smaller in MCs than in sTCs (two-sample $t$ tests, early $3 \mathrm{~Hz}$ MCs vs early $3 \mathrm{~Hz}$ sTCs, late $3 \mathrm{~Hz}$ MCs vs late $3 \mathrm{~Hz}$ sTCs, and so on; $p<0.05$ in all cases). Together, these 
A

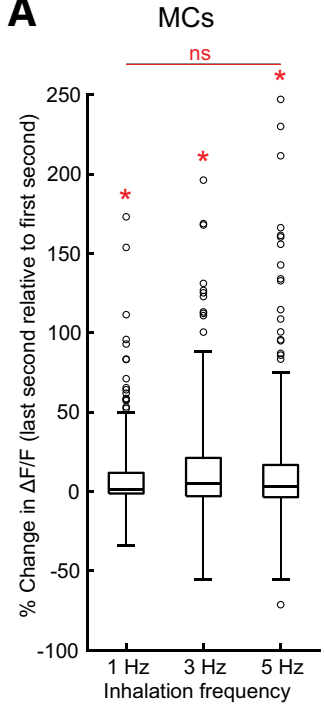

C Minus Strongly-

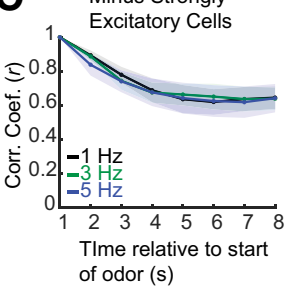

sTCs

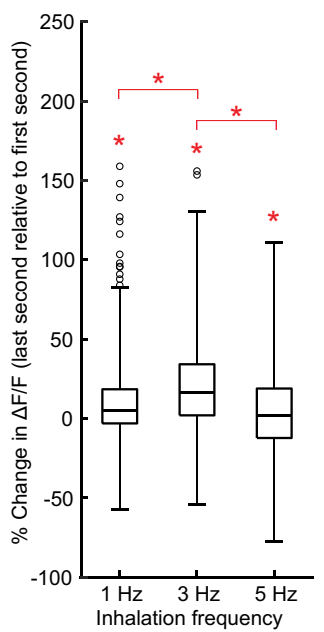

B

Change in MC Amplitude Change in MC Amplitude Relative to $1 \mathrm{~Hz}$, Early Relative to $1 \mathrm{~Hz}$, Late

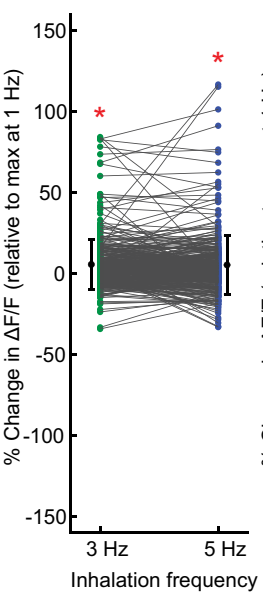

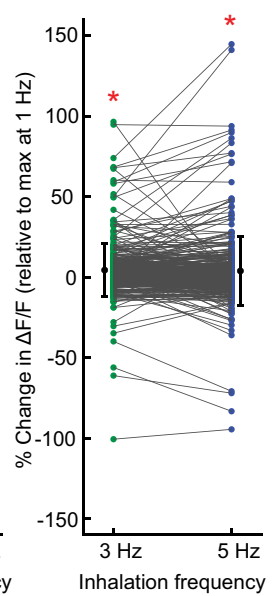

D Correlations in Final
sTCs

Change in sTC Amplitude Change in sTC Amplitude Relative to $1 \mathrm{~Hz}$, Early Relative to $1 \mathrm{~Hz}$, Late
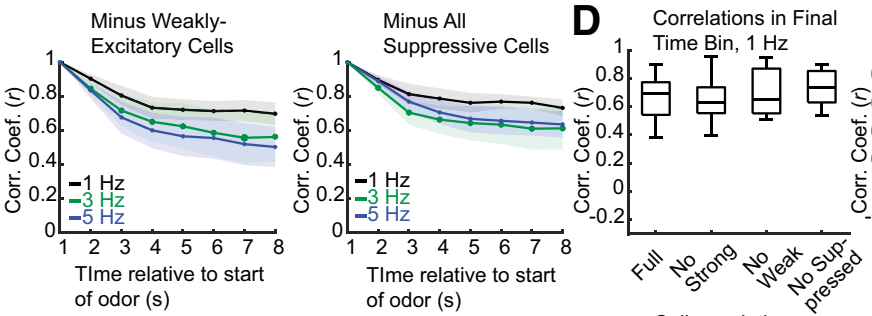

Cell population

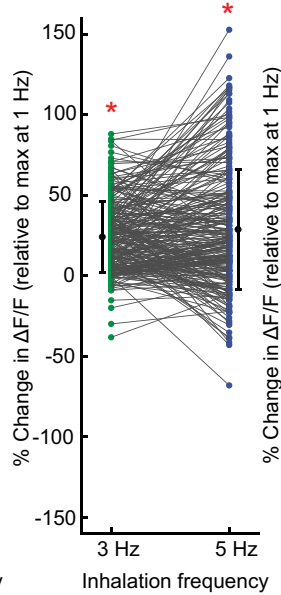

Correlations in Final

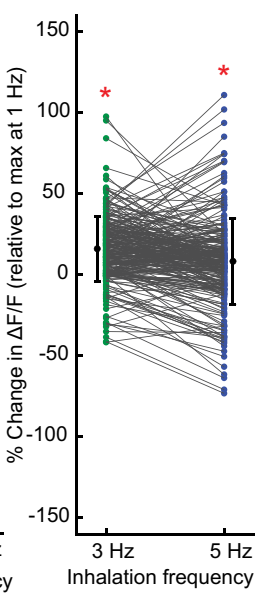

Correlations in Final

Time Bin, $3 \mathrm{~Hz} \quad 1$ Time Bin, $5 \mathrm{~Hz}$

Figure 4. $M C$ and TC responses show high variance in response to changing inhalation frequency. $A$, Change in signal amplitudes ( $\Delta \mathrm{F} / \mathrm{F}$ ) from the last second to the first second for all three inhalation frequencies across both cell types. In both MCs and sTCs, responses late in the odor presentation (final $1 \mathrm{~s}$ ) are slightly greater than responses early in the odor presentation (first 1 s), but mean changes are small relative to variance across cells. Boxplots represent means and 25th to 75th percentiles, and whiskers extend for 3 SDs. Outliers are plotted as individual points outside of the whiskers. $\boldsymbol{B}$, Change in strength of response magnitudes at 3 and $5 \mathrm{~Hz}$ compared with $1 \mathrm{~Hz}$, for both MCs (left) and sTCs (right). For both cell types, the plot on the left reflects change within the first second of odorant presentation, and the plot on the left shows change within the last second of odorant presentation. Points in each plot are relative to maximum value at $1 \mathrm{~Hz}$. For both MCs and sTCs, higher frequency inhalations lead to increased excitation (means in each plot are greater than zero). For MCs, early amplitudes change less than late amplitudes. The same holds for sTCs, although early amplitude changes are relatively much higher compared with MCs. Activity within the same cells are joined by lines. Beside the individual points, mean \pm SEM is plotted for each case. C, Correlation-over-time (Fig. 3C) for MCs, when a subpopulation of cells (as indicated by the titles of the figure panels) has been removed. Removing any subpopulation of cells still produces a high level of decorrelation, suggesting that no single group of cells dominates that population reformatting; ${ }^{*} p<0.01$ (see text for specific values). $\boldsymbol{D}$, Correlation-to-early values of the final time bins taken from $\boldsymbol{C}$, compared across all groups and inhalation-frequency conditions. No significant differences were found within any inhalation frequency.

results indicate that, while increasing inhalation frequency modestly enhances net excitation, repeated odorant sampling reorganizes MC and sTC responses differently. In particular, sTCs show an increase in excitation that affects all sTCs similarly, whereas MCs respond to increasing inhalation frequencies with a more modest increase in excitability, but more pronounced differentiation among individual cells.

Reformatting of the population responses among MCs could be a result of changes across all cells, or it could occur across only a subset of cells depending on their initial response to the odorant. To differentiate between these possibilities we broke the datasets into groups of strongly responding, weakly responding, and suppressive cells based on their response within the first second of odor presentation (see Materials and Methods); each group had roughly equivalent numbers of cells overall [average strongly-excited cells: $10(1 \mathrm{~Hz}), 10(3 \mathrm{~Hz}), 11(5 \mathrm{~Hz})$; weakly excited cells: $9(1 \mathrm{~Hz}), 10(3 \mathrm{~Hz}), 10(5 \mathrm{~Hz})$; suppressive cells: 9 $(1 \mathrm{~Hz}), 8(3 \mathrm{~Hz}), 7(5 \mathrm{~Hz}) ; 20$ odor-by-frequency presentations in seven mice]. To test whether any of these subpopulations preferentially drive reformatting, we removed each subset of cells and recalculated the correlation-to-early plots as done previously (compare with Fig. 3C). Removing strongly excited MCs from the population appeared to eliminate frequency-dependent differences in the degree of decorrelation (compare Figs. 4C, 3C). However, sampling-dependent decorrelation persisted after removal of each MC subgroup (strongly-excited, weakly-excited and suppressed MCs; Fig. 4C,D), with no significant differences in the degree of decorrelation relative to each other or relative to the full population, at any frequency (Kruskal-Wallis test: $1 \mathrm{~Hz}$, $\chi^{2}=0.72_{3,24}, p=0.87 ; 3 \mathrm{~Hz}, \chi^{2}=0.18_{3,24}, p=0.98 ; 3 \mathrm{~Hz}, \chi^{2}=$ $0.98_{3,24}, p=0.81$ ). Overall, these analyses show that decorrelation is not driven by changes in the responsiveness of particular subsets of MCs defined by their initial response; instead, all cells contribute to the population reformatting observed in our datasets.

Glomeruli are the functional unit of odor representations at the level of OB inputs, and each MC or TC has a single apical dendrite that branches heavily in one glomerulus and is the sole source of excitatory synaptic input for that cell (Mombaerts et al., 1996; Schoppa and Westbrook, 2001; Wachowiak et al., 2004; Wachowiak and Shipley, 2006; Economo et al., 2016). We have shown previously that temporally diverse response patterns, including suppressive and excitatory responses, are prevalent in glomerular MC signals (Economo et al., 2016). However, it is possible that different MCs arising from the same glomerulus (i.e., "sister" MCs; Dhawale et al., 2010) may show distinct effects 
A
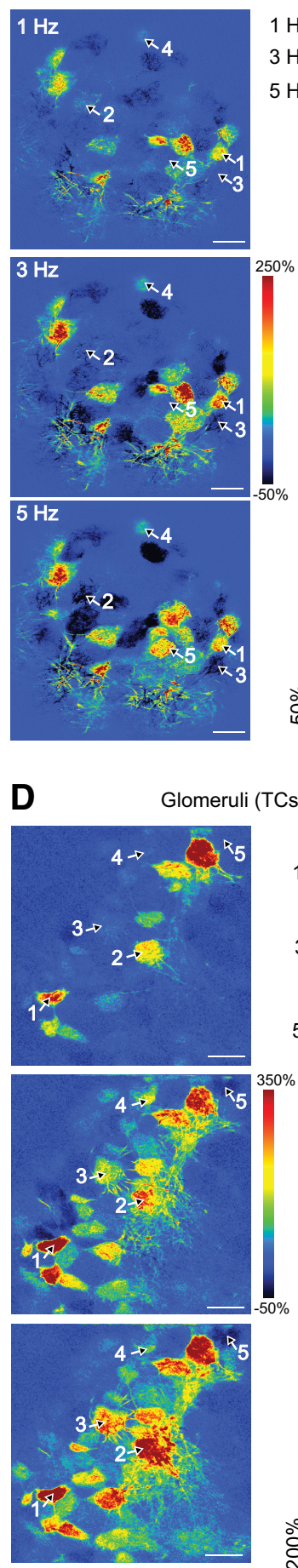

glom 1
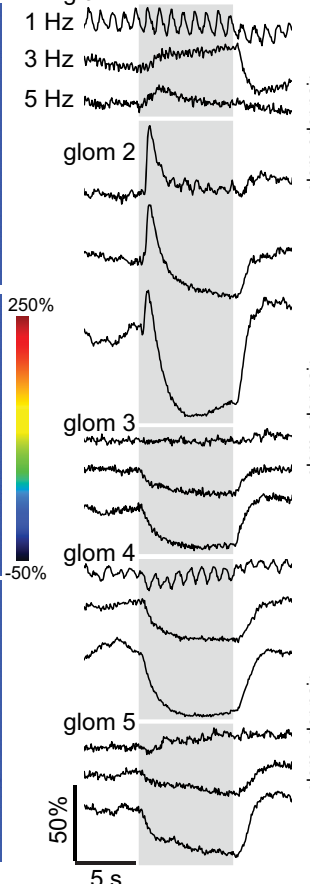

$5 \mathrm{~s}$

\section{Cs)}
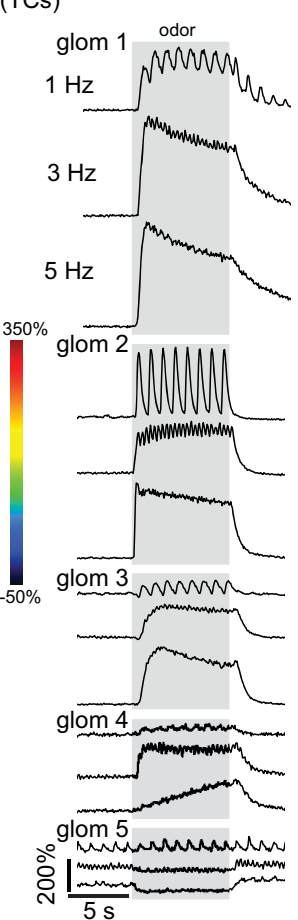

B Glomeruli (MCs)
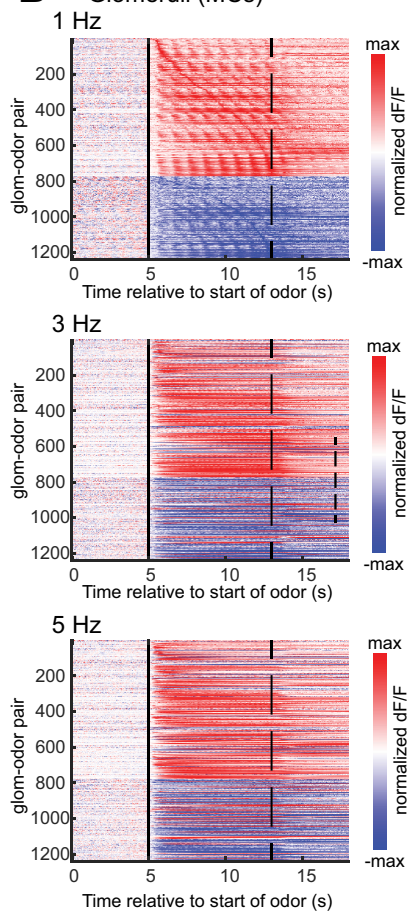

E

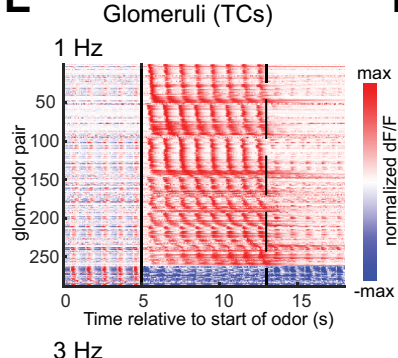

C Glomeruli (MCs)
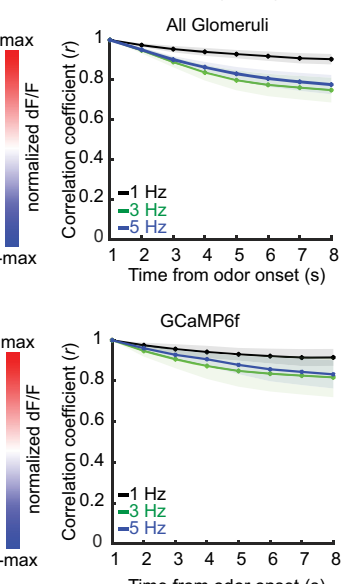

Time from odor onset (s)

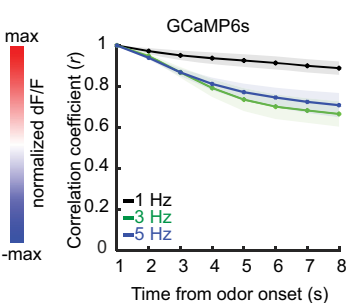

$\mathbf{F}$
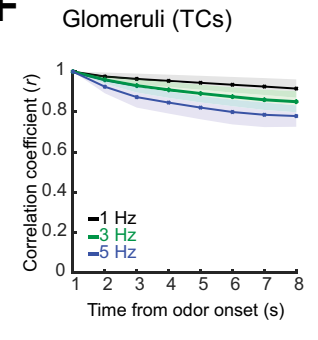
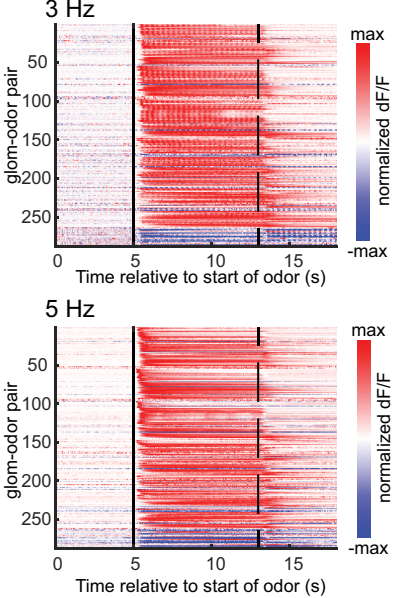

Figure 5. Glomerular signals from apical dendrites of MCs and sTCs show similar diversity in responses and decorrelation patterns compared with MC and sTC cell cell bodies. $A$, FOV of one experimental preparation, showing how the same set of MC glomeruli respond to three different inhalation frequencies of the same odorant. Right, Example trial-averaged responses from five glomeruli. Glomeruli have variable responses at differing inhalation frequencies. Glomerulus 2, for example, shows a quick burst of activity at all three frequencies, but at 3 and $5 \mathrm{~Hz}$, this initial burst is followed by a rapid decrease in activity below baseline, which is not seen at $1 \mathrm{~Hz}$. Glomerulus 4 shows slight excitation at $1 \mathrm{~Hz}$, and gradual suppression at 3 and $5 \mathrm{~Hz}$. Scale bars $=100 \mu \mathrm{m}$. $\boldsymbol{B}$, Time-by-activity plots for all MC glomeruli, rank-ordered by time-to-peak activity at $1 \mathrm{~Hz}$. Similar to MC cell bodies, glomerular responses are diverse and prolonged. MC glomeruli include a high proportion of suppressive responses. $C$, Correlation of the average MC glomerular population response to an odorant at a given time point to the population response at the first time point, over the length of odorant presentation. Top, All MC glomerular data. Bottom left, Data from GCaMP6f experiments. Bottom right, Data from GCaMP6s experiments. MC glomerular responses decorrelate more strongly at 3 and $5 \mathrm{~Hz}$ compared with $1 \mathrm{~Hz}$. The magnitude of decorrelation is lower than in MC-cell body data (compare to Fig. 3A). D, FOV of one TC experimental preparation, showing the same set of glomeruli responding to three different inhalation frequencies of the same odorant. Right, Example trial-averaged responses from five glomeruli. At low frequencies, fluorescence signals often follow inhalations, which is not seen at $5 \mathrm{~Hz}$. Glomeruli also show variable responses at differing inhalation frequencies. Glomerulus 1 , for example, shows inhalation-locked activity throughout 8-s odorant presentation, but at 3 and $5 \mathrm{~Hz}$, it instead shows a relatively quick burst of activity followed by slow adaptation throughout the remaining $8 \mathrm{~s}$, with a rapid drop-off when the odorant shuts off. Glomerulus 2 shows similar behavior to glomerulus 1 at $1 \mathrm{~Hz}$, but its response to 3 and $5 \mathrm{~Hz}$ differs. At $3 \mathrm{~Hz}$, it responds with high amplitude throughout the full 8-s presentation, but its response at $5 \mathrm{~Hz}$ instead shows a gradual adaptation. Scale bars $=100 \mu \mathrm{m}$. $\boldsymbol{E}$, Time-by-activity plots for all TC glomeruli, 
of repeated sampling or inhalation frequency, which could ultimately obscure the decorrelation among sister cells. Thus, to further investigate how inhalation frequency impacts odor representations, we imaged MC calcium signals from glomeruli of the dorsal OB.

We found that, as with cell body imaging, glomerular MC responses $(n=11$ mice, $15-41$ glomeruli per FOV) were more diverse at higher inhalation frequencies compared with low (Fig. $5 A, B)$. Suppressive responses were prevalent, occurring in 472 / $1164(40.5 \%)$ of all glomerulus-odorant pairs at $1-\mathrm{Hz}$ inhalation, with slightly lower percentages at high inhalation frequencies: $451 / 1164(38.7 \%)$ at $3 \mathrm{~Hz}$, and $391 / 1164(33.6 \%)$ at $5 \mathrm{~Hz}$. These percentages are similar to our MC-cell body data presented above, as well as to those reported earlier using a $2-\mathrm{Hz}$ inhalation frequency (Economo et al., 2016). We also observed frequencydependent changes in responsiveness as well as response polarity: from 1 to $3 \mathrm{~Hz}, 109 / 692$ (15.7\%) glomerulus-odor pairs switched from excited to suppressed, and from 1 to $5 \mathrm{~Hz}, 111 / 692$ (16.0\%) switched from excited to suppressed. Responses that switched from suppressed to excited were more common by a factor of 2: from 1 to $3 \mathrm{~Hz}, 114 / 472$ (24.2\%) switched from suppressed to excited, and from 1 to $5 \mathrm{~Hz}, 176 / 472$ (37.3\%) switched from suppressed to excited. These percentages are roughly similar to the percentages of MC cell bodies that switched polarity with increasing inhalation frequency.

Other aspects of the MC glomerular signals differed notably from the MC-cell body signals. For example, glomerular signals showed less decorrelation at the end of the $8 \mathrm{~s}$ odorant presentation compared with cell bodies ( $t$ test, $p=0.0037$; Fig. $5 C$ ), and they also showed a statistically significant effect of inhalation frequency on the amount of decorrelation, with responses at $1 \mathrm{~Hz}$ $(r=0.90)$ greater than those at $3 \mathrm{~Hz}(r=0.75$, Mann-Whitney $U$ test: $p=0.022, n=11$ mice $)$ and $5 \mathrm{~Hz}(r=0.78$; Mann-Whitney $U$ test: $p=0.036, n=11$ mice). Also, glomerular signals imaged with GCaMP6s appeared to show a greater difference in decorrelation between low and high inhalation frequencies compared with the GCaMP6f data (Fig. 5C). With both reporters, higher inhalation frequencies resulted in greater decorrelation, although only the result for GCaMP6s was significant (Kruskal-Wallis test: 6s, $\chi^{2}=6.86_{2,12}, p=0.032 ; 6 f, \chi^{2}=2.68_{2,15}, p=0.26$ ). Overall, these data point to a similar amount of decorrelation in MC dendrites as in their cell bodies.

We also analyzed glomerular signals among $\mathrm{CCK}^{+} \mathrm{TCs}$, to compare with response patterns recorded from sTC cell bodies. A caveat to this comparison is that the $\mathrm{CCK}^{+} \mathrm{TC}$ population also includes middle and deep TCs and, potentially, external TCs (Seroogy et al., 1985; Baltanás et al., 2011; Economo et al., 2016); thus, glomerular $\mathrm{CCK}^{+}$GCaMP6f signals cells may reflect summed responses across a more functionally diverse population than the sTC subtype. Glomerular TC responses showed some diversity in responses, but not as much as in MCs, as example traces show (Fig. 5D). Across the entire population of glomerulus-odor pairs ( $n=15-50$ per FOV, compiled from three mice), inhalation-linked activity was seen in 1 and $3 \mathrm{~Hz}$ conditions, and

\section{$\leftarrow$}

rank-ordered by time-to-peak activity at $1 \mathrm{~Hz}$. Signals follow individual inhalations at 1 and $3 \mathrm{~Hz}$. In general, responses last for the duration of odorant presentation, although especially at $5 \mathrm{~Hz}$, some cells can be seen to excite rapidly and adapt within the first $500 \mathrm{~ms}$ of odorant presentation. $\boldsymbol{F}$, Correlation of the average TC glomerular population response to an odorant at a given time point to the population response at the first time point, over the length of odorant presentation. The pattern and strength of decorrelation is very similar to that for STC cell bodies (Fig. 3D). most glomeruli showed sustained activity for the duration of the odorant presentation (Fig. 5E). Suppression was rare, occurring in $27 / 288(9.4 \%)$ of all glomerulus-odorant pairs at $1 \mathrm{~Hz}$ inhalation, with slightly greater percentages at high inhalation frequencies: $32 / 288(11.1 \%)$ at $3 \mathrm{~Hz}$ and $37 / 288(12.8 \%)$ at $5 \mathrm{~Hz}$. As with the sTC cell body imaging, $\mathrm{CCK}^{+}$glomerular signals showed little decorrelation from their initial response patterns with repeated sampling (Fig. $5 F$ ), with correlation coefficients after $8 \mathrm{~s}$ of odorant sampling of $r=0.92$ for $1 \mathrm{~Hz}$ inhalations, 0.85 at $3 \mathrm{~Hz}$, and $0.78 \mathrm{~Hz}$ at $5 \mathrm{~Hz}(n=288$ cell-odor pairs from three mice); these values were not significantly different from those observed among sTC cell bodies (comparing same inhalation frequencies, paired $t$ test, $p>0.05$ in all cases). These results suggest that dendritic signals from TCs behave in a similar fashion to sTC cell bodies.

Finally, we examined how the evolution of MC and sTC population responses might contribute to odor identity coding during repeated sampling. First, to better visualize how population responses change with repeated sampling, we performed PCA on the cell body population data (see Materials and Methods). Across all preparations, between $89.1 \%$ and $99.7 \%$ of the variance in temporal response was captured in the first three PCs. The high proportion of variance explained by only the first three PCs likely reflects simple but prevalent aspects of the odor-response time courses, such as slow increases in excitation or inhibition. Figure $6 A$ demonstrates this effect from an example MC dataset by displaying the change over time of odor responses in principal component space across the three inhalation frequencies. Odor representations grow further apart in the same $8 \mathrm{~s}$ block at higher inhalation frequencies compared with low (Fig. 6A). In contrast, the same plot in an example sTC imaging experiment (Fig. 6B) shows a quick evolution of the signal in PC space (top), followed by periodic "swings" in trajectories corresponding to responses to individual inhalations. This result is qualitatively similar to that seen in "synthetic" populations compiled from multiple MTC recordings (Bathellier et al., 2008).

To quantify these effects, we calculated the Euclidean distance between all cell responses in a FOV and averaged these across preparations (see Materials and Methods). At $1-\mathrm{Hz}$ inhalation, MC and sTC populations showed parallel trajectories in terms of the dynamics and magnitude of the change in Euclidean distance between different odor representations (Fig. 6C). However, at 3 and $5 \mathrm{~Hz}$ inhalation, MC populations diverge from sTC populations midway through the $8 \mathrm{~s}$ odor presentation, after which the distance between MC odor representations remains high, while that for sTC representations declined somewhat by the end of the $8 \mathrm{~s}$ odorant presentation, becoming more similar to the values at the beginning of the odorant presentation (Fig. 6D,E). Final Euclidean distance values between MC and sTC datasets were not statistically different at $1 \mathrm{~Hz}$ (MC mean $\pm \mathrm{SD}$ : $1.61 \pm 0.72$, sTC: $1.40 \pm 1.54$, paired $t$ test, $p=0.80)$, while the differences at 3 and $5 \mathrm{~Hz}$ were marginally significant $(3 \mathrm{~Hz}, \mathrm{MC}$ mean \pm SD: $2.81 \pm 1.05$, sTC: $1.41 \pm 0.90$, paired $t$ test, $p=0.10$; $5 \mathrm{~Hz}, \mathrm{MC}:$ mean $\pm \mathrm{SD}: 2.55 \pm 1.06$, sTC: $0.83 \pm 0.79$, paired $t$ test, $p=0.052$ ), owing to the high variability and relatively low sample sizes ( $n=5 \mathrm{MC}$ experiments and 3 sTC experiments). These differences in Euclidean distance between MCs and sTCs suggest that while sTC population responses to different odorants initially diverge, they fairly quickly plateau and, in many cases, begin to converge to be more similar within the period of odorant presentation. On the other hand, MC representations continue to diverge over the full $8 \mathrm{~s}$ odorant presentation and appear to diverge to a greater degree at the higher inhalation 
frequencies (Fig. 6C-E). Altogether, the results from the Euclidean distance analyses support a role for high-frequency inhalation in generating more distinct odor representations over the course of repeated sampling among MCs, with a smaller impact on odor representations among sTCs.

\section{Discussion}

Previous studies have demonstrated that a single inhalation of odorant is sufficient to robustly encode odorant identity, and that rodents can use information sampled in a single sniff to direct odor-guided behaviors (Rinberg et al., 2006; Kepecs et al., 2007; Wesson et al., 2008a,b). However, animals routinely sample odorants with bouts of high-frequency sniffing that can last multiple seconds (Vanderwolf, 1992; Thesen et al., 1993; Verhagen et al., 2007; Wesson et al., 2008a). Despite its being a fundamental aspect of active odor sampling, little is known about how such repeated sampling facilitates odor-guided behaviors or impacts odor representations. Earlier studies in diverse species have found that odor representations evolve with sustained odorant stimulation over a period of seconds (Friedrich and Laurent, 2001; Verhagen et al., 2007). Here, by imaging from many cells simultaneously and by targeting defined projection neuron subtypes, we have gained new insights into this process in the mammalian olfactory system. We found that repeated odorant sampling impacts odor representations by MCs and $\mathrm{CCK}^{+}$sTCs differently, with MCs showing a reformatting of the initial odor representation that increased in magnitude with increasing inhalation frequency, while the sTC odor representation remained relatively consistent across repeated samples and across frequencies. These results further support the idea that MCs and TCs constitute distinct functional pathways for early odor information processing, and suggest that these differences extend to the realm of repeated odorant sampling that is a hallmark of odor investigation in the behaving animal.

Imaging from relatively large numbers of cells simultaneously allowed us to gain insights into the nature of the reformatting of the MC population response. We observed that initial response magnitude had little influence in how MCs responded over time (Fig. 4C), instead, our results suggest that changes in response patterns across all cells contribute to the MC decorrelations. Our results are largely consistent with predictions from a previous study that relied on whole-cell recordings from presumptive MT cells (Díaz-Quesada et al., 2018), which found that individual MT cells showed diverse and frequency-dependent changes in excitation with repeated sampling at 1, 3, and $5 \mathrm{~Hz}$. Likewise, they are consistent with recordings of presumptive MT cells in awake mice, which found substantial changes in MT cell spiking patterns over the first few inhalations of odorant (Carey and Wachowiak, 2011; Patterson et al., 2013). The fact that we observed cell type-specific and frequency-dependent reformatting of MT cell odor representations that was sustained over a longer time period of up to $8 \mathrm{~s}$ in anesthetized mice suggests that the changes seen in awake mice likely result from 'bottom-up' effects related to changes in the dynamics of incoming sensory input, as opposed to modulation by centrifugal inputs related to behavioral state.

Our finding that MC responses evolved with repeated sampling over an $8 \mathrm{~s}$ odorant presentation substantially more than sTCs supports the idea that these two subpopulations serve distinct roles in odor information coding. In the context of active odor sampling, sTCs may convey a reliable, sniff-by-sniff snapshot of an animal's odor environment, while MCs may have the capacity to encode multiple aspects of odor information and do so flexibly, as a function of the animal's sampling behavior. MC activity patterns may also reflect distinct stimulus features at different times during a sniff bout, similar to what occurs in MCs of the zebrafish OB (Friedrich et al., 2004; Friedrich and Laurent, 2004), and relatedly, as occurs with repeated tastant sampling among neurons of gustatory cortex (Katz et al., 2001). 
The circuit mechanisms underlying the frequency-dependent reformatting of $\mathrm{MC}$ odor representations remain to be determined. Inhibition from granule cells onto MC lateral dendrites has been proposed to decorrelate sister MCs on a faster time scale (Dhawale et al., 2010), and this process may also work across repeated sampling. However, the fact that we observed similar, albeit somewhat smaller, time- and frequency-dependent changes in patterns of MC activity imaged across $\mathrm{OB}$ glomeruli, each of which contains the apical dendrites of multiple sister MCs, points to circuit mechanisms in the glomerular layer as playing an important role in this reformatting. One such mechanism may be interglomerular inhibitory circuits that are differentially engaged depending on the overall pattern of glomerular excitation relative to a given MC (Economo et al., 2016; DíazQuesada et al., 2018). TCs are thought to be less subject to this inhibition (Christie et al., 2001; Christie and Westbrook, 2006; Fukunaga et al., 2012; Phillips et al., 2012; Jordan et al., 2018b), consistent with our findings that these cells show more uniform responses over time and across inhalation frequency. Signals recorded from TC dendrites in the glomerular neuropil were generally similar to sTC cell body signals in their response patterns, although they showed a slightly greater divergence in decorrelation values across frequencies compared with the sTC cell body dataset. The similarity between these two datasets, despite the fact that sTCs comprise only a fraction of all TCs, is consistent with the idea that TCs with cell bodies deeper in the external plexiform layer are also functionally distinct from MCs (Fukunaga et al., 2012; Jordan et al., 2018b).

An important distinction in our comparison of sTCs and MCs is that we targeted sTCs defined by their expression of CCK, which constitutes a subset of the entire TC population (Seroogy et al., 1985; Liu and Shipley, 1994; Tobin et al., 2010). We have previously shown that $\mathrm{CCK}^{+}$sTCs show shorter-latency and simpler excitatory responses to odorants even relative to the larger TC population (Economo et al., 2016; Short and Wachowiak, 2019). This difference may explain why, in our previous study that relied simply on cell body depth to define MT subtype, we did not observe differences in prevalence of suppressive responses or impact of inhalation frequency on excitability of TCs and MCs (Díaz-Quesada et al., 2018). However, other studies have observed marked differences in MC and TC response dynamics using cell body depth as an identifying criterion (Fukunaga et al., 2012). Future experiments using additional genetic or anatomic criteria, for example, cortical projection target or expression of other transmitter receptors, will be important in better understanding the nature of diversity in the response properties of $\mathrm{OB}$ output neurons.

In behaving animals, inhalation frequencies occasionally reach $10 \mathrm{~Hz}$ or even higher, which is substantially faster than the highest frequency used here. However, even the $5 \mathrm{~Hz}$ inhalation frequencies used in this study, which approach technical limits of our artificial inhalation approach, show dramatic impacts on odor representations when contrasted with lower frequencies (Carey and Wachowiak, 2011; Díaz-Quesada et al., 2018). Additionally, while brief bouts of high-frequency inhalations may reach $10-12 \mathrm{~Hz}$, sustained bouts (lasting $2 \mathrm{~s}$ or more) rarely stay so high (Wesson et al., 2008a). At these higher frequencies, we might expect even greater decorrelation of initial response patterns, leading to rapid population-level reformatting of odorant responses. We also predict that such bouts could lead to higher proportions of suppressive responses in MCs, based on the modest increase in the prevalence of suppression seen when inhalation frequencies increased from 1 to $5 \mathrm{~Hz}$. Such a result could approach levels of suppression seen in awake mice (e.g., $46 \%$ in Kollo et al., 2014).

The diverse changes in MT cell excitability contrast with those of odorant responses among OSNs, where high-frequency inhalation more uniformly attenuates OSN inputs due to adaptation (Verhagen et al., 2007). How this adaptation leads to a more complex reformatting of MTC response patterns, including both decreases and increases in excitation, is unclear. Recently, by imaging glutamate signals onto MTC dendrites, we observed greater than expected diversity in the dynamics and magnitude of excitatory input onto MTCs during repeated sampling an anesthetized and awake mice (Moran et al., 2019). Thus, patterns of OSN-driven excitation onto MT cells may be more diverse than previously thought. In future experiments it will be important to image simultaneously from OSNs and MTCs to gain further insight into the mechanisms underlying this reorganization of odor representations as a function of sampling behavior.

\section{References}

Abraham NM, Spors H, Carleton A, Margrie TW, Kuner T, Schaefer AT (2004) Maintaining accuracy at the expense of speed: stimulus similarity defines odor discrimination time in mice. Neuron 44:865-876.

Baltanás FC, Curto GG, Gómez C, Díaz D, Murias AR, Crespo C, Erdelyi F, Szabó G, Alonso JR, Weruaga E (2011) Types of cholecystokinin-containing periglomerular cells in the mouse olfactory bulb. J Neurosci Res 89:35-43.

Bathellier B, Buhl DL, Accolla R, Carleton A (2008) Dynamic ensemble odor coding in the mammalian olfactory bulb: sensory information at different timescales. Neuron 57:586-598.

Carey RM, Wachowiak M (2011) Effect of sniffing on the temporal structure of mitral/tufted cell output from the olfactory bulb. J Neurosci 31:1061510626.

Christie J, Schoppa N, Westbrook G (2001) Tufted cell dendrodendritic inhibition in the olfactory bulb is dependent on NMDA receptor activity. J Neurophysiol 85:169-173.

Christie JM, Westbrook GL (2006) Lateral excitation within the olfactory bulb. J Neurosci 26:2269-2277.

Courtiol E, Hegoburu C, Litaudon P, Garcia S, Fourcaud-Trocmé N, Buonviso N (2011) Individual and synergistic effects of sniffing frequency and flow rate on olfactory bulb activity. J Neurophysiol 106:2813-2824.

Dhawale AK, Hagiwara A, Bhalla US, Murthy VN, Albeanu DF (2010) Nonredundant odor coding by sister mitral cells revealed by light addressable glomeruli in the mouse. Nat Neurosci 13:1404-1412.

Díaz-Quesada M, Youngstrom IA, Tsuno Y, Hansen KR, Economo MN, Wachowiak M (2018) Inhalation frequency controls reformatting of mitral/tufted cell odor representations in the olfactory bulb. J Neurosci 38:2189-2206.

Economo MN, Hansen KR, Wachowiak M (2016) Control of mitral/tufted cell output by selective inhibition among olfactory bulb glomeruli. Neuron 91:397-411.

Eiting TP, Wachowiak M (2018) Artificial inhalation protocol in adult mice. Bio Protoc 8:e3024.

Friedrich R, Habermann C, Laurent G (2004) Multiplexing using synchrony in the zebrafish olfactory bulb. Nat Neurosci 7:862-871.

Friedrich RW, Laurent G (2001) Dynamic optimization of odor representations by slow temporal patterning of mitral cell activity. Science 291:889894.

Friedrich RW, Laurent G (2004) Dynamics of olfactory bulb input and output activity during odor stimulation in zebrafish. J Neurophysiol 91:2658-2669.

Fukunaga I, Berning M, Kollo M, Schmaltz A, Schaefer Andreas T (2012) Two distinct channels of olfactory bulb output. Neuron 75:320-329.

Gong S, Zheng C, Doughty ML, Losos K, Didkovsky N, Schambra UB, Nowak NJ, Joyner A, Leblanc G, Hatten ME, Heintz N (2003) A gene expression atlas of the central nervous system based on bacterial artificial chromosomes. Nature 425:917-925.

Griff ER, Mafhouz M, Chaput MA (2008) Comparison of identified mitral and tufted cells in freely breathing rats: II. Odor-evoked responses. Chem Senses 33:793-802. 
Haddad R, Lanjuin A, Madisen L, Zeng H, Murthy VN, Uchida N (2013) Olfactory cortical neurons read out a relative time code in the olfactory bulb. Nat Neurosci 16:949-957.

Jordan R, Kollo M, Schaefer AT (2018a) Sniffing fast: paradoxical effects on odor concentration discrimination at the levels of olfactory bulb output and behavior. eNeuro 5 .

Jordan R, Fukunaga I, Kollo M, Schaefer AT (2018b) Active sampling state dynamically enhances olfactory bulb odor representation. Neuron 98:1214-1228.e5.

Katz DB, Simon SA, Nicolelis MA (2001) Dynamic and multimodal responses of gustatory cortical neurons in awake rats. J Neurosci 21: 4478-4489.

Kepecs A, Uchida N, Mainen ZF (2007) Rapid and precise control of sniffing during olfactory discrimination in rats. J Neurophysiol 98:205-213.

Kollo M, Schmaltz A, Abdelhamid M, Fukunaga I, Schaefer AT (2014) 'Silent' mitral cells dominate odor responses in the olfactory bulb of awake mice. Nat Neurosci 17:1313-1315.

Laska M (1990) Olfactory sensitivity to food odor components in the shorttailed fruit bat, Carollia perspicillata (Phyllostomatidae, Chiroptera). J Comp Physiol A Neuroethol Sens Neural Behav Physiol 166:395-399.

Liu WL, Shipley MT (1994) Intrabulbar associational system in the rat olfactory bulb comprises cholecystokinin-containing tufted cells that synapse onto the dendrites of GABAergic granule cells. J Comp Neurol 346:541558.

Madisen L, Garner AR, Shimaoka D, Chuong AS, Klapoetke NC, Li L, van der Bourg A, Niino Y, Egolf L, Monetti C, Gu H, Mills M, Cheng A, Tasic B, Nguyen TN, Sunkin SM, Benucci A, Nagy A, Miyawaki A, Helmchen F, et al. (2015) Transgenic mice for intersectional targeting of neural sensors and effectors with high specificity and performance. Neuron 85:942-958.

Mombaerts P, Wang F, Dulac C, Chao SK, Nemes A, Mendelsohn M, Edmondson J, Axel R (1996) Visualizing an olfactory sensory map. Cell 87:675-686.

Moran AK, Eiting TP, Wachowiak M (2019) Diverse dynamics of glutamatergic input underlie heterogeneous response patterns of olfactory bulb mitral and tufted cells in vivo. bioRxiv 692574. doi: 10.1101/692574.

Nagayama S, Takahashi YK, Yoshihara Y, Mori K (2004) Mitral and tufted cells differ in the decoding manner of odor maps in the rat olfactory bulb. J Neurophysiol 91:2532-2540.

Patterson MA, Lagier S, Carleton A (2013) Odor representations in the olfactory bulb evolve after the first breath and persist as an odor afterimage. Proc Natl Acad Sci USA 110:E3340-E3349.

Phillips ME, Sachdev RNS, Willhite DC, Shepherd GM (2012) Respiration drives network activity and modulates synaptic and circuit processing of lateral inhibition in the olfactory bulb. J Neurosci 32:85-98.

Rinberg D, Koulakov A, Gelperin A (2006) Speed-accuracy tradeoff in olfaction. Neuron 51:351-358.

Rothermel M, Brunert D, Zabawa C, Díaz-Quesada M, Wachowiak M (2013) Transgene expression in target-defined neuron populations mediated by retrograde infection with adeno-associated viral vectors. J Neurosci 33:15195-15206

Rygg AD, Van Valkenburgh B, Craven BA (2017) The influence of sniffing on airflow and odorant deposition in the canine nasal cavity. Chem Senses 42:683-698.
Schoppa NE, Westbrook GL (2001) Glomerulus-specific synchronization of mitral cells in the olfactory bulb. Neuron 31:639-651.

Seroogy KB, Brecha N, Gall C (1985) Distribution of cholecystokinin-like immunoreactivity in the rat main olfactory bulb. J Comp Neurol 239:373383.

Short SM, Wachowiak M (2019) Temporal dynamics of inhalation-linked activity across defined subpopulations of mouse olfactory bulb neurons imaged in vivo. eNeuro 6 .

Taniguchi H, He M, Wu P, Kim S, Paik R, Sugino K, Kvitsiani D, Kvitsani D, Fu Y, Lu J, Lin Y, Miyoshi G, Shima Y, Fishell G, Nelson SB, Huang ZJ (2011) A resource of Cre driver lines for genetic targeting of GABAergic neurons in cerebral cortex. Neuron 71:995-1013.

Thesen A, Steen JB, Døving KB (1993) Behaviour of dogs during olfactory tracking. J Exp Biol 180:247-251

Tobin VA, Hashimoto H, Wacker DW, Takayanagi Y, Langnaese $\mathrm{K}$ Caquineau C, Noack J, Landgraf R, Onaka T, Leng G, Meddle SL, Engelmann M, Ludwig M (2010) An intrinsic vasopressin system in the olfactory bulb is involved in social recognition. Nature 464:413-417.

Uchida N, Mainen ZF (2003) Speed and accuracy of olfactory discrimination in the rat. Nat Neurosci 6:1224-1229.

Uchida N, Kepecs A, Mainen ZF (2006) Seeing at a glance, smelling in a whiff: rapid forms of perceptual decision making. Nat Rev Neurosci 7:485-491.

Vanderwolf CH (1992) Hippocampal activity, olfaction, and sniffing: an olfactory input to the dentate gyrus. Brain Res 593:197-208.

Verhagen JV, Wesson DW, Netoff TI, White JA, Wachowiak M (2007) Sniffing controls an adaptive filter of sensory input to the olfactory bulb. Nat Neurosci 10:631-639.

Wachowiak M (2011) All in a sniff: olfaction as a model for active sensing. Neuron 71:962-973

Wachowiak M, Cohen LB (2001) Representation of odorants by receptor neuron input to the mouse olfactory bulb. Neuron 32:723-735.

Wachowiak M, Shipley MT (2006) Coding and synaptic processing of sensory information in the glomerular layer of the olfactory bulb. Semin Cell Dev Biol 17:411-423.

Wachowiak M, Denk W, Friedrich RW (2004) Functional organization of sensory input to the olfactory bulb glomerulus analyzed by two-photon calcium imaging. Proc Natl Acad Sci USA 101:9097-9102.

Wachowiak M, Economo MN, Díaz-Quesada M, Brunert D, Wesson DW, White JA, Rothermel M (2013) Optical dissection of odor information processing in vivo using GCaMPs expressed in specified cell types of the olfactory bulb. J Neurosci 33:5285-5300.

Welker WI (1964) Analysis of sniffing in the albino rat. Behavior 22:223-244.

Wesson DW, Donahou TN, Johnson MO, Wachowiak M (2008a) Sniffing behavior of mice during performance in odor-guided tasks. Chem Senses 33:581-596.

Wesson DW, Carey RM, Verhagen JV, Wachowiak M (2008b) Rapid encoding and perception of novel odors in the rat. PLoS Biol 6:e82.

Youngentob SL, Mozell MM, Sheehe PR, Hornung DE (1987) A quantitative analysis of sniffing strategies in rats performing odor detection tasks. Physiol Behav 41:59-69.

Zhao K, Dalton P, Yang GC, Scherer PW (2006) Numerical modeling of turbulent and laminar airflow and odorant transport during sniffing in the human and rat nose. Chem Senses 31:107-118. 